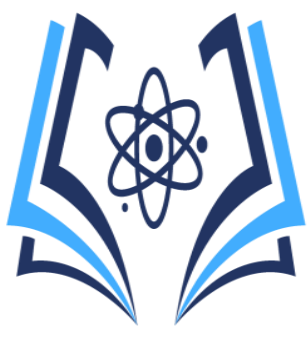

\section{Rol de la enfermera en los pacientes con complicaciones postoperatorias gastrointestinales}

\author{
Role of the nurse in patients with postoperative \\ gastrointestinal complications
}

\section{Rol de la enfermera en los pacientes}

\author{
Lic. Susana Delgado Bernal Mg \\ Ab. Sidar Solórzano Solórzano Mg. ${ }^{2}$ \\ ${ }^{1}$ Universidad Estatal del Sur de Manabí, delgado.susana@hotmail.com, orcid.org/0000-0002-1081-2614 \\ ${ }^{2}$ Universidad Estatal del Sur de Manabí, sidarsolorzano@ hotmail.com, orcid.org/0000-0002.6950-8397
}


HIGIA DE LA SALUD

and warmth, it will be an effective tool in nursing practice based on both the Virginia Henderson 14 needs model, the PAE and the taxonomies NANDA, NIC and NOC.

Key Words: surgical, quality of life, immediate postoperative period, care guide.

\section{Introducción}

Las complicaciones postoperatorias son eventos frecuentes, particularmente en los pacientes de alto riesgo; no solo tienen repercusión clínica en el período postoperatorio inmediato, sino también a largo plazo, con afectación de la calidad de vida e incremento en la mortalidad. Muchos de los pacientes con estas características requieren admisión en las Unidades de Cuidados Intensivos (UCI) para un mejor control y manejo de los desórdenes fisiopatológicos agudos. Las complicaciones postoperatorias más frecuentes son: obstrucción intestinal, hemorragia digestiva, diarrea, íleo paralitico, fistula y complicaciones propias del estoma. (1)

Existen una serie de complicaciones postoperatorias que puede presentar el paciente luego de ser sometido a una cirugía, es por ello que en la etapa posquirúrgica, la atención de la enfermera debe estar centrada, en torno a la valoración integral del paciente y su vigilancia continua, por lo cual se deben implementar medidas de prevención y cuidados de enfermería, centrados en los sistemas que más se vieron afectados, con el propósito de proporcionar una asistencia de alta calidad profesional, y lograr así la recuperación óptima del paciente, en función de su estado y potencial de recuperación.

Por ello, el presente estudio es de suma importancia, ya que será una fuente de información relevante sobre las complicaciones postoperatorias gastrointestinales y de las intervenciones de enfermería, que se deben llevar a cabo con el fin de lograr resultados en la evolución del paciente. Lo cual será beneficioso tanto para los estudiantes y profesionales de enfermería, como para los pacientes y sus familiares, ya que incorporaran nuevos conocimientos, que les permitirá llevar un mejor manejo de los cuidados en el postoperatorio, favorecer la reducción de la morbimortalidad e incide directamente en mejorar la calidad de vida de la población.

Según datos estadísticos, la obstrucción intestinal reporta a nivel mundial el $15 \%$ de todas las
Periodo. Enero - Junio 2020

Vol. 2, Nro. 1, Publicado 2020-06-30

consultas vista en un servicio de emergencias por dolor abdominal, cerca de 300mil admisiones hospitalarias anuales en los Estados Unidos y con cifras de mortalidad que van desde un $2 \%$ hasta un $30 \%$. Estudios realizados en Cuba, describen cifras de mortalidad que oscilan entre en $10 \mathrm{y}$ el $20 \%$, con una tasa de mortalidad de un $4 \%$ (2). En cuanto a la falla anastomótica es una de las principales complicaciones de las cirugías gastrointestinales, que se presenta en 3,4 a $15 \%$ de los pacientes y las ostomías son un procedimiento quirúrgico que ha aumentado en los últimos años y alcanza un $65 \%$ de aplicación y tiene un índice de mortalidad del 7\% (3).

Mientras que el Íleo paralitico en el postoperatorio, es un problema médico importante dado que es la causa más frecuente de retardo en el egreso de los pacientes, después de una cirugía presenta una incidencia que se sitúa entre un $0,5 \%$ y un $23 \%$ de los pacientes, es su incidencia variable en función del tipo de intervención quirúrgica realizada (4)

En Ecuador un estudio realizado en el Hospital Metropolitano de Quito sobre obstrucción intestinal en el postoperatorio se estima que se presenta en un 55,47\% de los pacientes; ocupando la décima causa de muerte dentro de población general (5). Las ostomías de acuerdo a un estudio realizado por Meneses Valeria y Miranda Ausay a cerca de las complicaciones del estoma en el 2019 presenta una frecuencia del $23 \%$, en cuanto al íleo paralítico de acuerdo a un estudio realizado en el Hospital del Niño Dr. Francisco de Icaza Bustamante se ha observado casos de en pacientes pediátricos de cuatro a cinco años con el $40 \%$ de incidencia para este grupo etario (6)

Materiales y métodos:

El proyecto integrador de saberes, es de tipo descriptiva - analítica basada en la revisión bibliográfica sobre rol de enfermería en los pacientes con complicaciones postoperatorias gastrointestinales, con el fin de recopilar la información más relevante sobre el estado actual del tema. Se logró identificar los problemas y/o necesidades de salud que lo afectan, con los datos obtenidos se formularon los diagnósticos de enfermería y una guía de cuidados para dar paso al segundo momento de la investigación.

\section{Resultados}

Incidencia de las complicaciones postoperatorias gastrointestinales en Ecuador

\begin{tabular}{ll} 
Complicaciones & Incidencia \\
\hline Hemorragia gastrointestinal & $26,70 \%$ \\
\hline Íleo paralitico & $15,60 \%$ \\
\hline
\end{tabular}


Obstrucción intestinal

Guía de cuidados de enfermería en pacientes con complicaciones postoperatorias gastrointestinales

\section{Introducción}

Las Guías de Atención de Enfermería contienen el conjunto de recomendaciones e indicaciones necesarias para la estandarización de los procesos de atención, factor que hace falta para contribuir a la calidad de la atención sanitaria. Son realizadas a través de la búsqueda bibliográfica de la información científica y la adaptación local de la misma, la cual será utilizada como instrumento de apoyo y de consulta permite maximizar la calidad de la atención, así como también ayudará en la identificación oportuna de signos y síntomas de potenciales complicaciones en este periodo (7).

Este material se ha elaborado con el objetivo de mejorar la práctica asistencial en el posoperatorio, y disminuir la incidencia de las complicaciones gastrointestinales en el postoperatorio. En su contenido se consideran las etapas del Proceso de Atención de Enfermería, como son la valoración que se evidenciada en los datos objetivos: el diagnóstico de enfermería, las intervenciones y finalmente la evaluación. Como también encontrará explicaciones sobre los cuidados que se deben tener después de la operación. Esta información le ayudará en su posterior recuperación (8).

\section{Población objetivo}

Profesionales de enfermería.

\section{Objetivo}

- Mejorar la práctica asistencial en el posoperatorio y disminuir la incidencia de las complicaciones gastrointestinales en el postoperatorio.

\section{Justificación}

Siendo la obstrucción intestinal, las complicaciones del estoma, el íleo paralitico, la diarrea, las complicaciones gastrointestinales más frecuentes en el postoperatorias de acuerdo con los artículos citados, es necesario identificar las intervenciones adecuadas para poder responder de forma eficiente y rápida ante estas complicaciones. Por lo que esta guía, ha sido elaborada como una necesidad para modificar la situación existente, y para facilitar el trabajo del profesional de enfermería. La cual tiene como objetivo mejorar el bienestar físico y emocional del paciente, mediante una oportuna intervención, lo cual se logrará al seguir cada una de las diversas intervenciones que se describen en la guía.

\section{Personal responsable}

Licenciada en enfermería Equipo

- Colchonetas

- Equipo para aspiración de secreciones

- Equipo para administración de oxigeno

- Monitor multiusos de signos vitales

- Estetoscopio y termómetro

Equipo accesorio o de apoyo

- Oxímetros

- Bisturí

- Camilla

Materiales

- Jeringas

- Guantes

- Semiluna

- Solución salina

- Gasas o apósitos

- Equipo de venoclisis

- Catéteres

- Tijeras

- Medicación indicada

PAE en pacientes con complicaciones postoperatorias gastrointestinales

\section{Procedimiento}

Valoración

Se realiza la valoración al paciente en la Unidad de Reanimación Pos Anestésica (URPA) por lo que es un lugar donde el enfermo pasa relativamente poco tiempo, en primer lugar, se tomará las constantes vitales cada 15 minutos y nos centraremos en las necesidades más importantes en esta etapa de su evolución, para la realización del mismo se tomará en cuenta el modelo de las 14 necesidades básicas de Virginia Henderson debido a que es un modelo que utiliza un lenguaje sencillo y fácilmente comprensible. Además, expone de manera clara, el rol autónomo de la enfermera y la especificidad de su servicio en la atención sanitaria.

- Temperatura

- Eliminación

- Seguridad

- Alimentación / Hidratación

- Reposo / sueño

- Respiración

- Comunicación

- Movilización

- Higiene/piel

- Religión/creencias

- Trabajar/realizarse

- Actividades lucidas 


\section{HIGÍA DE LA SALUD}

- Aprender

- Vestirse

Se valorará al paciente con las diferentes escalas que se utilizan en el área de URPA:

- Escala de Alderete

- Escala de Bromage

- Escala del dolor
Periodo. Enero - Junio 2020

Vol. 2, Nro. 1, Publicado 2020-06-30

- Escala de Morse - Downton.

Plan de cuidados postoperatorio inmediato y mediato. De acuerdo, con los resultados encontrados y a los datos objetivos se establece el siguiente plan de cuidados, y aclara que los resultados que se obtengan en la evaluación, es lo que se desea conseguir.

\section{a. Temperatura}

\begin{tabular}{|c|c|c|c|c|}
\hline DX & PLANIFICACIÓN & & EYE & \\
\hline NANDA & NOC & NIC & EJECUCIÓN & EVALUACIÓN \\
\hline $\begin{array}{l}\text { 00005: Riesgo de } \\
\text { desequilibrio de la } \\
\text { temperatura corporal } \\
\text { R/C } \\
\text { Enfermedad } \\
\text { traumatismo que afecta } \\
\text { la regulación de la } \\
\text { temperatura } \\
\text { M/P } \\
\text { Hipotermia } \\
\text { Hipertermia } \\
\text { Escalofríos }\end{array}$ & $\begin{array}{l}\text { 602: Hidratación } \\
\text { 800: Termorregulación } \\
\text { 1902: Control del } \\
\text { riesgo }\end{array}$ & $\begin{array}{l}\text { 3900: Regulación de } \\
\text { la temperatura } \\
\text { 4120: Manejo de } \\
\text { líquidos } \\
\text { 6680: Monitorización } \\
\text { de los signos vitales }\end{array}$ & $\begin{array}{l}\text { Se realizan las } \\
\text { actividades que están } \\
\text { basadas en cada una de } \\
\text { las intervenciones } \\
\text { establecidas esperando } \\
\text { una raspuesta } \\
\text { satisfactoria en cuanto } \\
\text { a la temperatura. }\end{array}$ & $\begin{array}{l}\text { El paciente } \\
\text { mantiene una } \\
\text { temperatura } \\
\text { corporal que se } \\
\text { encuentra dentro } \\
\text { de los niveles } \\
\text { establecidos. }\end{array}$ \\
\hline $\begin{array}{lc}\text { Actividades }-\mathbf{3 9 0 0} \\
- & \text { Comprobar la tem } \\
- & \text { Observar color y te } \\
- & \text { Enseñar al pacient } \\
& \text { Utilizar colchón té } \\
- & \text { Ajustar la tempera } \\
- & \text { Administrar la me } \\
- & \text { Administrar antipi } \\
- & \text { Utilizar colchón re }\end{array}$ & $\begin{array}{l}\text { nperatura de la piel. } \\
\text { a evitar el agotamiento p } \\
\text { mico y mantas calientes p } \\
\text { rra ambiental a las necesi } \\
\text { icación adecuada para evi } \\
\text { cticos, si está indicado. } \\
\text { rigerante y baños tibios pa }\end{array}$ & $\begin{array}{l}\text { tiempo indicados. } \\
\text { r el calor y los golpes d } \\
\text { ra ajustar la temperatur } \\
\text { ades del paciente. } \\
\text { ar o controlar los escalo } \\
\text { ra ajustar la temperatura }\end{array}$ & $\begin{array}{l}\text { calor } \\
\text { corporal alterada, si proc } \\
\text { ríos. } \\
\text { corporal adecuada, si pro }\end{array}$ & ede. \\
\hline $\begin{array}{ll}\text { Actividades - 4120 } \\
- & \text { Pesar y controlar } \\
- & \text { Realizar un registr } \\
- & \text { Realizar sondaje } \\
- & \text { Observar si hay in } \\
- & \text { Administrar líquid } \\
- & \text { Administrar los di } \\
- & \text { Instruir al paciente }\end{array}$ & $\begin{array}{l}\text { evolución. } \\
\text { preciso de ingesta y elim } \\
\text { sical, si es preciso. } \\
\text { icios de sobrecarga / reten } \\
\text { s, si procede. } \\
\text { réticos prescritos, si proce } \\
\text { en el estado de nada por b }\end{array}$ & $\begin{array}{l}\text { nación. } \\
\text { ción de líquidos } \\
\text { le. }\end{array}$ & & \\
\hline \begin{tabular}{ll}
\multicolumn{2}{l}{ Actividades -6680} \\
- & Valorar signos vita \\
- & Observar y registra \\
- & Observar periódica \\
- & Observar si el paci \\
- & Observar si hay lle
\end{tabular} & $\begin{array}{l}\text { es, si procede. } \\
\text { si hay signos y síntomas } \\
\text { nente el color, la tempera } \\
\text { nte presenta cianosis cent } \\
\text { ado capilar normal. }\end{array}$ & $\begin{array}{l}\text { le hipotermia e hiperter } \\
\text { ura y la humedad de la } \\
\text { al y periférica. }\end{array}$ & & \\
\hline
\end{tabular}

\section{b. Eliminación}




\begin{tabular}{|c|c|c|c|c|}
\hline \multirow{2}{*}{$\begin{array}{l}\text { DX } \\
\text { NANDA }\end{array}$} & \multicolumn{2}{|l|}{ PLANIFICACIÓN } & \multicolumn{2}{|l|}{ E Y E } \\
\hline & NOC & NIC & EJECUCIÓN & EVALUACIÓN \\
\hline $\begin{array}{l}\text { 00013 Diarrea } \\
\text { R/C Efectos adversos } \\
\text { de los medicamentos. } \\
\text { Infección } \\
\text { M/P Eliminación por } \\
\text { lo menos de tres } \\
\text { deposiciones líquidas } \\
\text { por día. } \\
\text { 00011 Estreñimiento } \\
\text { R/C Dolor abdominal } \\
\text { Infección } \\
\text { M/P Eliminación de } \\
\text { heces duras, secas y } \\
\text { formadas, sangre roja } \\
\text { brillante en las heces, } \\
\text { heces negras } \\
\text { alquitranadas, } \\
\text { distención abdominal. }\end{array}$ & $\begin{array}{l}\text { 501: Eliminación } \\
\text { intestinal } \\
\text { 602: Hidratación } \\
\text { 208: Nivel de } \\
\text { movilidad } \\
\text { 1800: Estado } \\
\text { nutricional: } \\
\text { ingestión } \\
\text { alimentaria y de } \\
\text { líquidos }\end{array}$ & $\begin{array}{l}\text { 460: Manejo de la } \\
\text { diarrea } \\
\text { 430: } \\
\text { intestinal } \\
\text { 450: Manejo } \\
\text { estreñimiento de } \\
\text { impactación. }\end{array}$ & $\begin{array}{lr}\text { Se realizan } & \text { las } \\
\text { actividades } & \\
\text { planteadas en } & \text { cada } \\
\text { una de } & \text { las } \\
\text { intervenciones } & \\
\text { establecidas, } & \\
\text { esperando } & \text { una } \\
\text { respuesta } & \\
\text { satisfactoria } & \text { con } \\
\text { respecto a } & \text { la } \\
\text { eliminación. } & \end{array}$ & $\begin{array}{l}\text { El paciente realiza } \\
\text { deposiciones normales. }\end{array}$ \\
\hline 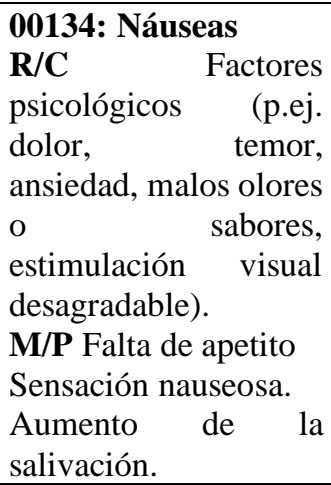 & $\begin{array}{l}\text { 2103: Gravedad } \\
\text { del síntoma }\end{array}$ & $\begin{array}{l}\text { 1450: Manejo de las } \\
\text { náuseas } \\
\text { 1570: Manejo del } \\
\text { vómito } \\
\begin{array}{l}\text { 2380: Manejo de } \\
\text { medicación }\end{array}\end{array}$ & $\begin{array}{l}\text { 1450: Manejo de las } \\
\text { náuseas } \\
\text { 1570: Manejo del } \\
\text { vómito } \\
\begin{array}{l}\text { 2380: Manejo de } \\
\text { medicación }\end{array}\end{array}$ & $\begin{array}{l}\text { El paciente no presenta } \\
\text { sensación nauseosa ni } \\
\text { vómito gracias a los } \\
\text { valores de electrolitos } \\
\text { normales. }\end{array}$ \\
\hline \multicolumn{5}{|c|}{$\begin{array}{l}\text { Actividades - } \mathbf{4 6 0} \\
\text { - } \\
\text { - }\end{array}$} \\
\hline \multicolumn{5}{|c|}{$\begin{array}{l}\text { Actividades - } \mathbf{4 3 0} \text { y } \mathbf{4 5 0} \\
\text { - } \quad \text { Controlar los movimientos intestinales, incluyendo la frecuencia, consistencia, forma, volumen y color, si procede. } \\
\text { - }\end{array}$} \\
\hline
\end{tabular}


- Fomentar el aumento de la ingesta de líquidos, a menos que esté contraindicado.

- Enseñar al paciente / familia a mantener un diario de comidas.

- Instruir al paciente / familia acerca de la dieta rica en fibras, si procede.

- Administrar el enema o la irrigación, cuando proceda.

- Pesar al paciente regularmente.

\section{Actividades - 1450}

- Realizar una valoración completa de las náuseas, incluyendo la frecuencia, duración, intensidad y los factores desencadenantes, utilizando herramientas como Diario de autocuidado, Escala analógica visual, Escala descriptiva de Duke e índice de Rhodes de náuseas y vómitos.

- Reducir o eliminar los factores personales que desencadenan o aumentan las náuseas (ansiedad, miedo, fatiga, y ausencia de conocimientos).

- Fomentar el descanso y el sueño adecuados para facilitar el alivio de las náuseas.

- Pesar al paciente con regularidad.

- Utilizar una higiene bucal frecuente para fomentar la comodidad, a menos que eso estimule las náuseas.

- Verificar los efectos de las náuseas.

\section{Actividades - 1570}

- Valorar el color, consistencia, presencia de sangre, duración, frecuencia y alcance de emesis.

- Medir o estimar el volumen de la emesis.

- Reducir o eliminar los factores personales que desencadenen o aumenten los vómitos.

- Colocar al paciente de forma adecuada para prevenir la aspiración.

- Mantener las vías aéreas abiertas.

- $\quad$ Proporcionar apoyo físico durante el vómito

- Utilizar higiene oral para limpiar boca y nariz.

- Esperar 30 min., después del vómito para dar líquidos (dando por sentado tracto gastrointestinal y peristaltismo normal).

- Controlar el equilibrio de fluidos y de electrolitos.

- Fomentar el descanso.

- Pesar al paciente con regularidad.

- Controlar los efectos del control del vómito.

\section{Actividades - 2380}

- Observar los efectos terapéuticos de la medicación en el paciente.

- Observar si hay signos y síntomas de toxicidad de la medicación.

- Vigilar los niveles de suero en sangre (electrolitos, protrombina, medicamentos), si procede.

- Revisar periódicamente con el paciente y/o familia, los tipos y dosis de medicamentos tomados.

- Explicar al paciente y/o la familia la acción y los efectos secundarios esperados de la medicación

\begin{tabular}{|c|c|c|c|c|}
\hline \multicolumn{5}{|c|}{ c. Seguridad } \\
\hline DX & PLANIFICACIĆ & & E Y E & \\
\hline NANDA & NOC & NIC & EJECUCIÓN & EVALUACIÓN \\
\hline $\begin{array}{l}\text { 00155 Riesgo de } \\
\text { caídas } \\
\text { R/C Dificultad } \\
\text { en la marcha. } \\
\text { Administración } \\
\text { de narcóticos. } \\
\text { M/P } \\
\text { Debilidad } \\
\text { Mareo }\end{array}$ & $\begin{array}{l}\text { 1902: Control del } \\
\text { riesgo } \\
\text { 1909: Conducta } \\
\text { de seguridad: } \\
\text { prevención de } \\
\text { caídas }\end{array}$ & $\begin{array}{l}\text { 6490: } \\
\text { Prevención de } \\
\text { caídas } \\
\text { 6654: Vigilancia: } \\
\text { seguridad }\end{array}$ & $\begin{array}{l}\text { Se realizan las } \\
\text { actividades que están } \\
\text { basadas en cada una } \\
\text { de las intervenciones } \\
\text { establecidas } \\
\text { esperando una } \\
\text { respuesta satisfactoria } \\
\text { en cuanto a la }\end{array}$ & $\begin{array}{l}\text { El paciente puede movilizarse } \\
\text { con ayuda sin ningún problema. }\end{array}$ \\
\hline
\end{tabular}


ISSN 2773-7705

HIGÍA DE LA SALUD

Periodo. Enero - Junio 2020

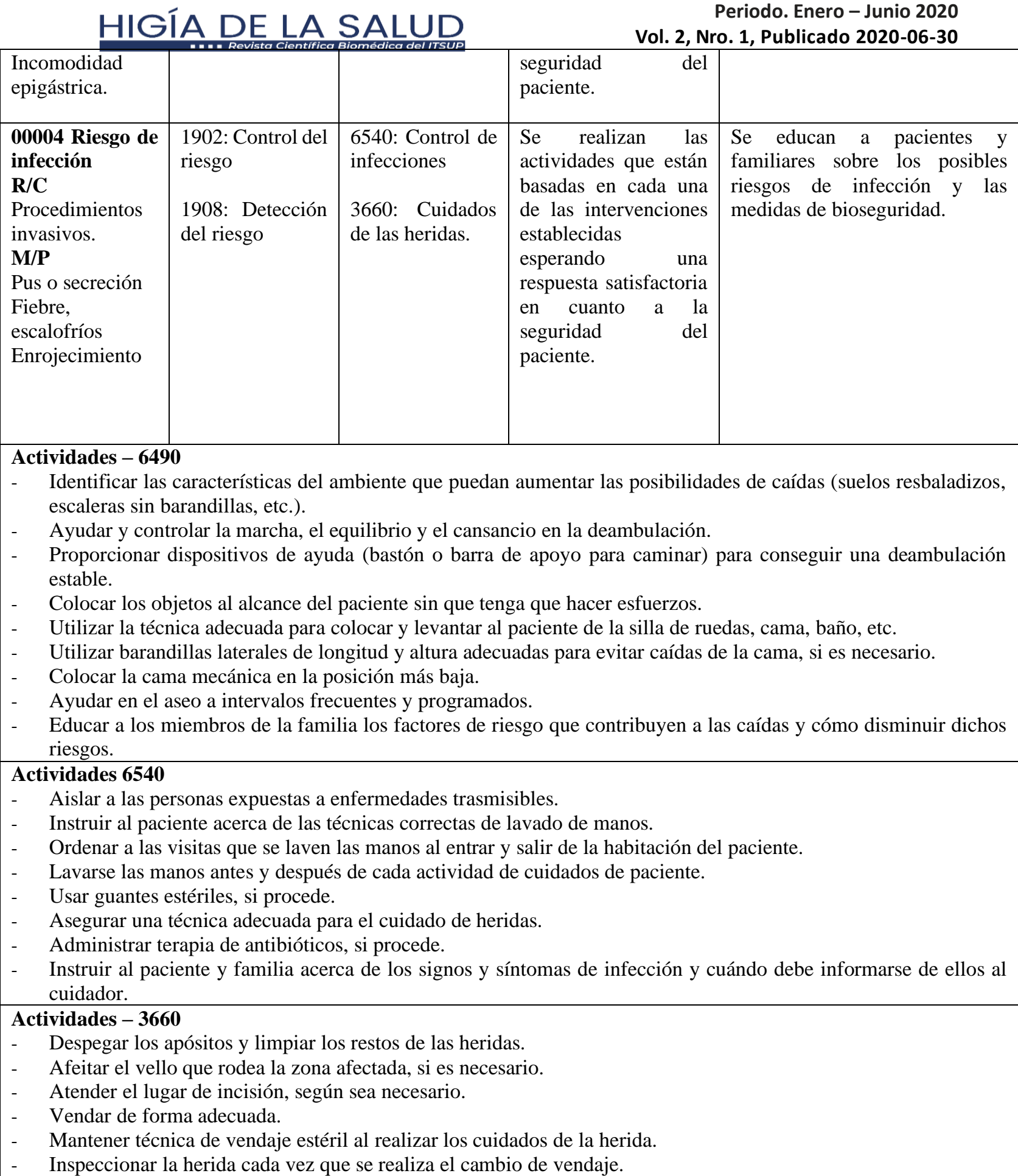

\section{Dieta}

Se inicia la ingesta oral dentro de las primeras 24 horas después del procedimiento, siempre que puedan deglutir normalmente, con indicación escrita de inicio de la vía oral y que no tengan alteraciones en la función o integridad intestinal; ya que es efectivo para mejorar la evolución clínica en la etapa postoperatoria. Luego de tolerar la dieta de líquidos claros, progresar a dieta blanda, ya que es efectivo para evitar la malnutrición.

Los alimentos no deben contener irritantes, picantes o condimentos en exceso, debe ser moderada en grasas, hay que emplear formas sencillas de preparación de los alimentos (cocidos, hervidos, etc.), se debe evitar los alimentos fritos o muy salados y las salsas, ya que pueden causar molestias.

Comidas Día $1 \quad$ Día 2
principales




\begin{tabular}{|c|c|c|c|}
\hline \multicolumn{4}{|c|}{ Vol. 2, Nro. 1, Publicado 2020-06-30 } \\
\hline Desayuno & $\begin{array}{l}1 \text { taza de té de } \\
\text { manzanilla }+1 \text { taza de } \\
\text { Atol de avena }+1 \text { pera } \\
\text { mediana sin piel y } \\
\text { cocida }\end{array}$ & $\begin{array}{l}\text { Pan blanco de molde con } 1 \\
\text { rebanada de queso blanco }+ \\
1 \text { vaso de jugo de manzana }\end{array}$ & $\begin{array}{l}1 \text { taza de té }+1 \text { huevo duro }+ \\
\text { rebanada de queso blanco }+1 \\
\text { manzana pequeña sin piel y } \\
\text { cocida }\end{array}$ \\
\hline $\begin{array}{l}\text { Merienda de } \\
\text { la mañana }\end{array}$ & $\begin{array}{l}1 \text { taza de té de } \\
\text { manzanilla con } 3 \\
\text { galletas María }\end{array}$ & 1 vaso de jugo de durazno & 1 taza de gelatina \\
\hline $\begin{array}{l}\text { Almuerzo/ } \\
\text { Cena }\end{array}$ & $\begin{array}{l}\text { Caldo de pollo con papa } \\
\text { y zanahoria }\end{array}$ & $\begin{array}{l}90 \text { grs de Pechuga de pollo } \\
\text { en tiras con puré de papas, } \\
\text { acompañada de ensalada } \\
\text { cocida de zanahoria. }\end{array}$ & $\begin{array}{l}90 \text { grs de pavo desmenuzado } \\
\text { con puré de calabaza, } \\
\text { acompañada de ensalada } \\
\text { cocida de berenjena con } \\
\text { zanahoria. }\end{array}$ \\
\hline $\begin{array}{l}\text { Merienda de } \\
\text { la tarde }\end{array}$ & $\begin{array}{l}1 \text { manzana mediana sin } \\
\text { cáscara y cocida }\end{array}$ & $\begin{array}{l}1 \text { taza de té con } 3 \text { galletas } \\
\text { María }\end{array}$ & $\begin{array}{l}1 \text { pera } \\
\text { mediana sin cáscara cocida }\end{array}$ \\
\hline
\end{tabular}

\section{Recomendaciones dietéticas}

- Se deben evitar los alimentos ricos en grasas como snacks, embutidos, frituras, mantequilla, salsas y alimentos industrializados ricos en azúcares, debido a que estos alimentos son proinflamatorios dificultado el proceso de cicatrización, así como la digestión (9)

- Deben evitarse alimentos que puedan irritar la mucosa intestinal, como es el caso de los picantes, pimienta y bebidas ricas en cafeína, así como alimentos ricos en fibras, ya que su absorción a nivel intestinal es más lenta y promueve un aumento en el tamaño de las heces, debiendo evitar vegetales y frutas

\section{Conclusiones}

- Los factores de riesgo asociados a las complicaciones postoperatorias gastrointestinales son numerosos. y estos dependen a diferentes causas como, el tipo de procedimiento quirúrgico al que se vaya a someter, así como también de las enfermedades crónicas y malos hábitos alimenticios.

- Las complicaciones post operatorias gastrointestinales con mayor frecuencia a nivel del Ecuador, de acuerdo con los diversos estudios citados son: la obstrucción intestinal, las complicaciones del estoma y la hemorragia digestiva.

\section{Colaboradores en la investigación:}

Bravo Alcívar Jhon Alejandro, Chancay Segura Marian Michelle, Coaboy Romero María Belén, Fortis García Jaritza Geraldine, García Kelal Axel Joseve, Hidalgo Lucas Juliana Maite, Játiva Lino Karina Lisbeth, Menéndez Zambrano Johana Jailene, Morales Reyes Jean Pierre.

\section{Bibliografía}

1. Martos-Benítez FD, Gutiérrez-Noyola A, Echevarría-Víctores A. Postoperative complications and crudas y con cáscara, alimentos integrales y frutos secos (10)

- Evitar los alimentos que favorecen la producción de gases intestinales como los frijoles, el repollo, los brócolis, los espárragos, las coles, entre otros, también deben ser evitados, debido a que pueden causar malestar y dolor (11)

- Seleccione alimentos de origen animal bajo en grasa.

- Elija grasas saludables.

- Coma menos azúcares.

- Ingerir entre 1,5 a 2 litros de agua al día para prevenir el estreñimiento y para reducir la presión abdominal que se ejerce al evacuar.

- Realizar actividad física todos los días.

- La guía de cuidados de enfermería para las complicaciones postoperatorias gastrointestinales, permitirá a los enfermeros ofrecer cuidados adecuados, para aquellos pacientes que lleguen a presentar este tipo de complicaciones, así como también les permitirá reducir el nivel de incidencia en el postoperatorio. Esta guía será una herramienta eficaz en la práctica enfermera, especialmente en el posoperatorio bajo el fundamento tanto del modelo de las 14 necesidades de Virginia Henderson, el PAE y las taxonomías NANDA, NIC Y NOC. 
clinical outcomes among patients undergoing thoracic and gastrointestinal cancer surgery: A prospective cohort study. Rev Bras Ter Intensiva [Internet]. 2016 Jan 1 [cited 2020 Sep 7];28(1):40-8. Available from: http://www.scielo.br/scielo.php?script=sci_arttext\&pid=S0103$507 X 2016000100040 \& \operatorname{lng}=\mathrm{en} \& \mathrm{nrm}=\mathrm{iso} \& \operatorname{lng}=\mathrm{es}$.

2. Alexander Marchena RodríguezI RJP, JCSS. Morbimortalidad en pacientes operados por oclusión intestinal en el Servicio de Cirugía General: cifras alarmantes [Internet]. 2015 [cited 2020 Sep 4]. Available from: http://scielo.sld.cu/scielo.php?script=sci_arttext\&pid=S222124342015000100004

3. Maikel Adolfo Pacheco, Guillermo Eduardo Aldana, Luis Eduardo Martínez, Juan Carlos Forero, Carlos Andrés Gómez, Esteban Mauricio Coral, et al. Incidencia de falla anastomótica en intestino delgado, colon y recto, Bogotá, Colombia [Internet]. 2017 [cited 2020 Sep 4]. Available from: http://www.scielo.org.co/pdf/rcci/v32n4/v32n4a5.pdf

4. Regalado AB, Luis J, Cabrera AMC, Fernando B, Cajamarca -Perú M. "Factores De Riesgo Asociados A Íleo Postoperatorio Prolongado En Intervenciones Quirúrgicas Abdominales En El Servicio De Cirugía Del Hospital General De Cajamarca En El Periodo Febrero De 2016 A Febrero De 2017. 2016.

5. Guasti Sinaluisa Paola Estefanía. Prevalencia De Pacientes Con Obstrucción Intestinal Resueltos Clínicamente Versus Resueltos Quirúrgicamente Que Acuden Al Hospital General De Latacunga . 2018.

6. Erick Ricardo Pérez. Causas De Ileoparalitico En Niños De 4 A 10 Años Y Su Manejo Quirúrgico. Hospital Francisco De Icaza Bustamante, Año 2015 A 2018 [Internet]. 2019 [cited 2020 Sep 4]. Available from: http://repositorio.ug.edu.ec/bitstream/redug/43422/1/CD 2984- PEREZ DÍAZ\%2C ERICK RICARDO.pdf

7. De G, De Enfermeria C. Hospital María Auxiliadora Departamento De Enfermeria Servicio De Cirugia. 2012.

8. Guía de Práctica Clínica sobre Cuidados Perioperatorios en Cirugía Mayor Abdominal [Internet]. 2016 [cited 2020 Aug 21]. Available from: https://ulcerasfora.sergas.gal/Informacion/Documents/201/cuidados-perioperatorios-cirugia.pdf

9. $\triangleright$ Dieta tras extirpación de la vesícula biliar [Internet]. [cited 2020 Aug 31]. Available from: https://www.dietfarma.com/dietas/dieta-tras-extirpacion-de-la-vesicula-biliar

10. Dieta baja en fibra y residuos: MedlinePlus enciclopedia médica [Internet]. [cited 2020 Sep 5]. Available from: https://medlineplus.gov/spanish/ency/patientinstructions/000200.htm

11. Qué comer después de una apendicitis (con menú ejemplo) - Tua Saúde [Internet]. [cited 2020 Aug 31]. Available from: https://www.tuasaude.com/es/que-comer-despues-de-una-apendicitis/ 